OPEN ACCESS

Edited by:

Jatinder K. Lamba,

University of Florida, United States

Reviewed by:

Rossana Roncato,

Aviano Oncology Reference Center

(IRCCS), Italy

Ruty Mehrian-Shai,

Sheba Medical Center, Israel

*Correspondence:

Giorgia Marisi

giorgia.marisi@irst.emr.it

Specialty section:

This article was submitted to

Pharmacogenetics and

Pharmacogenomics,

a section of the journal

Frontiers in Pharmacology

Received: 22 July 2021

Accepted: 19 October 2021

Published: 10 November 2021

Citation:

Ulivi P, Passardi A, Marisi G, Chiadini E, Molinari C, Canale M, Pasini L, Ferroni F, Frassineti GL, Bartolini G and Monti M (2021) Case Report: The Added Value of Liquid Biopsy in Advanced Colorectal Cancer From

Clinical Case Experiences.

Front. Pharmacol. 12:745701.

doi: 10.3389/fphar.2021.745701

\section{Case Report: The Added Value of Liquid Biopsy in Advanced Colorectal Cancer From Clinical Case Experiences}

\author{
Paola Ulivi ${ }^{1}$, Alessandro Passardi ${ }^{2}$, Giorgia Marisi ${ }^{1 *}$, Elisa Chiadini ${ }^{1}$, Chiara Molinari ${ }^{1}$, \\ Matteo Canale ${ }^{1}$, Luigi Pasini ${ }^{1}$, Fabio Ferroni ${ }^{3}$, Giovanni Luca Frassineti ${ }^{2}$, Giulia Bartolini ${ }^{2}$ and \\ Manlio Monti ${ }^{2}$
}

${ }^{1}$ Biosciences Laboratory, IRCCS Istituto Romagnolo per lo Studio dei Tumori (IRST) "Dino Amadori", Meldola (FC), Italy, ${ }^{2}$ Medical Oncology Unit, IRCCS Istituto Romagnolo per lo Studio dei Tumori (IRST) "Dino Amadori", Meldola (FC), Italy, ${ }^{3}$ Radiology Unit, IRCCS Istituto Romagnolo per lo Studio dei Tumori (IRST) "Dino Amadori", Meldola (FC), Italy

Liquid biopsy represents a valid strategy for tumor molecular characterization. It gives the opportunity to bypass tumor heterogeneity, to monitor tumor characteristics during the course of treatment, and to perform the analysis even when tumor tissue is not available or inadequate. In the clinical practice of metastatic colorectal cancer, tumor molecular characterization is crucial for patient management, as RAS and BRAF status could influence the treatment choice. Although for this type of cancer tumor tissue is usually available at diagnosis, liquid biopsy could give complementary information and could permit monitoring of the mutation status during the course of treatment. At present, there are no clinical indications for its use in clinical practice. However, we report four clinical cases for which liquid biopsy analysis gave integrative information with respect to tumor tissue characterization, which permits us to understand the unresponsiveness of patients to treatment, with potential implications in patient's management.

Keywords: CtDNA, metastatic colorectal cancer, RAS, mutations, case report

\section{INTRODUCTION}

Accurate tumor molecular characterization is fundamental for targeted therapy and precision medicine. For advanced colorectal cancer (CRC), therapeutic agents against the epidermal growth factor receptor (EGFR) represent the standard of treatment for those patients carrying a wild type RAS status (Di Fiore et al., 2007; Karapetis et al., 2008). Usually, tumor tissue derived from surgery or endoscopic procedures represents the gold standard specimens for RAS molecular characterization. Contrary to other solid tumors, for which very often the tumor tissue available is very scarce and insufficient for the molecular determinations, for CRC the tumor material is usually enough to permit the performing of all the necessary molecular analyses. However, tumors are known to have spatial and temporal heterogeneity, and mutations not identified in primary tumors have been shown to be present in the metastatic lesions (Knijn et al., 2011; Furuki et al., 2018). Moreover, treatment could induce clonal evolution with the consequent acquisition of further molecular alteration (Misale et al., 2012; Yamada et al., 2016). Liquid biopsy, mainly in terms of circulating tumor DNA (ctDNA), represents a valid option to study the molecular characteristics of tumors, bypassing the issue of spatial heterogeneity and permitting the monitoring of the clonal evolution during treatment (Diehl et al., 2008; Murtaza et al., 2013). A recent study has demonstrated 
August 2018

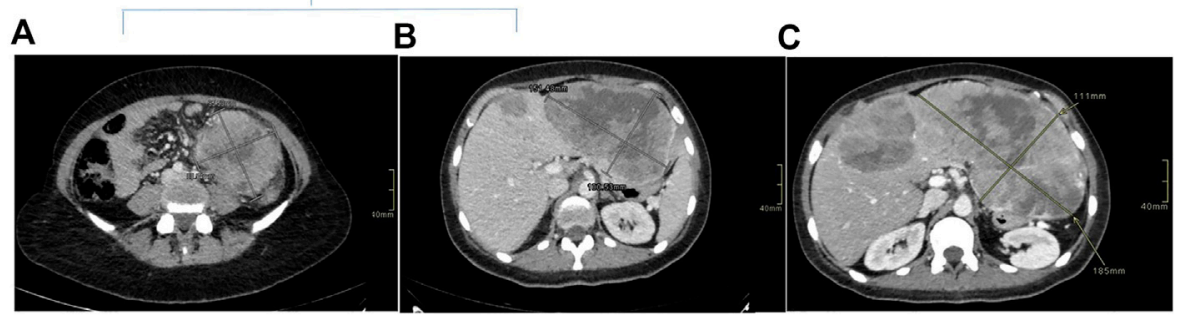

FIGURE 1 | Contrast enhanced CT scan demonstrating descending colon cancer at baseline (A) and liver metastasis at baseline (B) and at progression (C).

that the use of liquid biopsy could complement that of tumor tissue, showing that the combined use of the approaches could increase the diagnostic accuracy (Takeda et al., 2019).

To date, however, the use of liquid biopsy is not recommended in clinical practice for patients with advanced CRC, and it is performed only sporadically based on the Medical Oncologist's request.

Here we present the history of four patients with advanced CRC, all with a baseline tumor tissue characterization revealing a $R A S$ and $B R A F$ wild type (wt) status and receiving an anti-EGFR treatment that was resistant to treatment. In all four patients, the liquid biopsy analysis revealed important information not evidenced by tumor tissue analysis.

\section{CASE PRESENTATIONS}

\section{Case A}

Towards mid-2018, a 40- to 45-year-old patient underwent colonoscopy because of persistent constipation, with a diagnosis of stenotic colorectal adenocarcinoma on splenic flexure. The chest and abdominal CT scan showed a metastatic disease, in particular a lesion on the left lobe of the liver of about $107 \times 67 \times 94 \mathrm{~mm}$ and six lesions on the right lobe, the bigger of which was about $21 \mathrm{~mm}$ (Figure 1A,B). There was also peritoneal carcinomatosis. At diagnosis CEA was $1.8 \mu \mathrm{g} / \mathrm{L}$ $(<5 \mu \mathrm{g} / \mathrm{L})$ and $\mathrm{Ca} 19-914.5 \mathrm{KU} / \mathrm{L}(<37 \mathrm{KU} / \mathrm{L})$. The molecular characterization was performed on the primary tumor tissue using a MassARRAY Sequenom analysis (Myriapod Colon status, Diatech Pharmacogenetics, Jesi, Italy), revealing a $R A S$ and $B R A F$ wt status. Consequently, the patient received a firstline treatment with FOLFOXIRI (Irinotecan $150 \mathrm{mg} / \mathrm{mq}$ over $1 \mathrm{~h}$ day 1 , oxaliplatin $85 \mathrm{mg} / \mathrm{mq}$ over $2 \mathrm{~h}$ day 1 , calciolevofolinate $200 \mathrm{mg} / \mathrm{mq}$ over $2 \mathrm{~h}$ day 1,5 fluorouracil $2400 \mathrm{mg} / \mathrm{mq}$ over $48 \mathrm{~h}$ ) plus Panitumumab (6 mg/kg over $1 \mathrm{~h}$ day 1$)$ every 2 weeks. After four cycles of treatment, CT scan evaluation showed disease progression, in particular on the left lobe of the liver where the bigger metastasis was $180 \mathrm{~mm}$ (Figure 1C), while there was an improvement on the primary tumor because the colorectal cancer step from $96 \times 89 \mathrm{~mm}$ to $61 \times 54 \mathrm{~mm}$. Because of the young age of the patient, a liquid biopsy sample was made to obtain useful information for new drugs. RAS and $B R A F$ status were analyzed by a Real-Time PCR method (Easy-KRAS status, Easy-NRAS status, Easy-BRAF status, Diatech Pharmacogenetics). Results showed the presence of a BRAF mutation (V600E). Concomitantly, the patient had a performance deterioration with severe abdominal pain and received a second-line treatment with FOLFIRI (Irinotecan $180 \mathrm{mg} / \mathrm{mq}$ over $90 \mathrm{~min}$ day 1 , calciolevofolinate $200 \mathrm{mg} / \mathrm{mq}$ over $2 \mathrm{~h}$ day $1-2,5$ fluorouracil $400 \mathrm{mg} / \mathrm{mq} 3^{\prime}-4^{\prime}$ day 1 , 5 fluorouracil $2,400 \mathrm{mg} / \mathrm{mq}$ $48 \mathrm{~h}$ day 1 ) plus aflibercet $(4 \mathrm{mg} / \mathrm{kg}$ over $1 \mathrm{~h})$ every 2 weeks. Unfortunately, the patient rapidly progressed with a decline of performance status and died after 1 month from chemotherapy. Based on the results obtained on liquid biopsy about V600E $B R A F$ mutation, the same analysis was repeated on DNA of the tumor tissue using a more sensitive methodology (Real-Time PCR), but a wt $B R A F$ status was confirmed. These results suggest two hypotheses: 1) $B R A F$ mutation was already present in the primary tumor tissue but heterogeneously, and tumor tissue analysis was not able to evidence the mutation; 2) $B R A F$ mutation was induced during treatment as a secondary resistance mechanism.

\section{Case B}

At the end of 2014, a 55 to 60-year-old patient underwent pancolonscopy because of rectal bleeding, with a diagnosis of rectal adenocarcinoma placed at $8,9 \mathrm{~cm}$ from anal rhyme. The abdominal and chest CT scan showed a localized disease on the proximal rectum. The pelvic NMR described a rectal cancer and the disease was a cT3N + M0. At diagnosis, CEA was $10.1 \mu \mathrm{g} / \mathrm{L}$ $(<5 \mu \mathrm{g} / \mathrm{L})$. The patient received a neo-adjuvant treatment inside a clinical trial, with FOLFOX4 (Oxaliplatin $85 \mathrm{mg} / \mathrm{mq}$ over $2 \mathrm{~h}$ day 1 , calciolevofolinate $100 \mathrm{mg} / \mathrm{mq}$ over $2 \mathrm{~h}$ day $1-2,5$ fluorouracil $400 \mathrm{mg} / \mathrm{mq}^{\prime}{ }^{\prime}-4^{\prime}$ day $1-2,5$ fluorouracil $600 \mathrm{mg} / \mathrm{mq} 22 \mathrm{~h}$ day $1-2$ every 2 weeks) for 4 cycles and hypofractionated radiotherapy ( $25 \mathrm{~Gy} / 5 \mathrm{fr}$ ) that was administered between the second and the third cycle of chemotherapy, After the neoadjuvant treatment CEA was $5.7 \mu \mathrm{g} / \mathrm{Land}$ the patient underwent a surgical resection of rectum (ypT2N1cR0). After surgery, the patient performed an adjuvant therapy with FOLFOX4 for eight cycles and at the end of therapy, CEA was $4.8 \mu \mathrm{g} / \mathrm{L}$. In early 2017 disease relapse was shown, with bilaterally pulmonary metastases at CT scan and CEA increased to $8.7 \mu \mathrm{g} / \mathrm{L}$ (Figure 2).

Molecular characterization performed using MassARRAY Sequenom (Myriapod Colon status, Diatech Pharmacogenetics) on the tumor tissue of the surgical resection of 2015 showed a BRAF and 


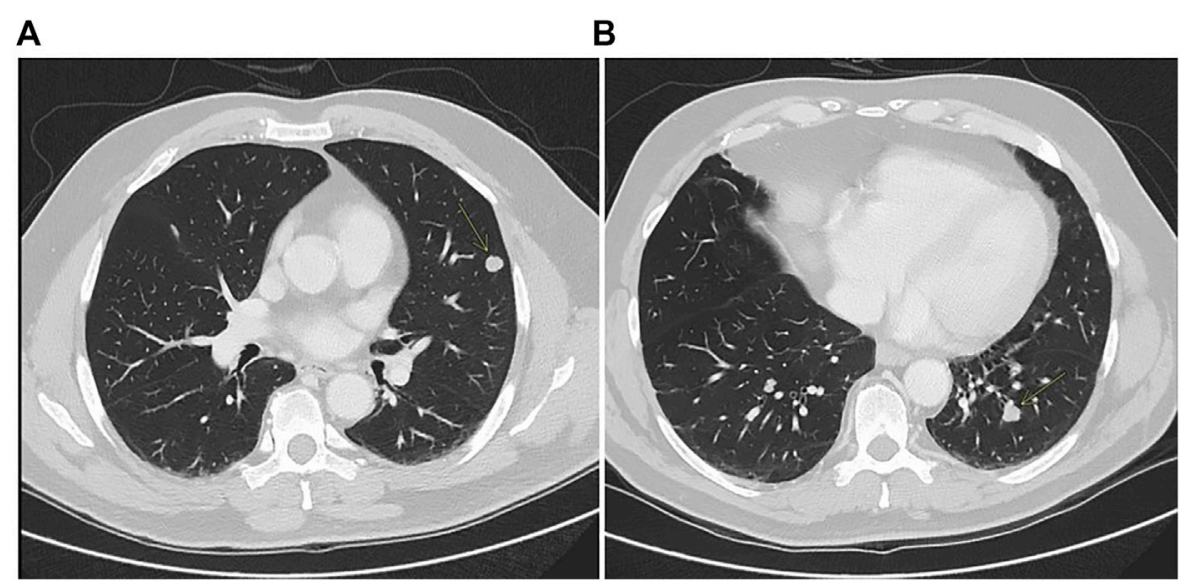

FIGURE 2 | CT scan showing left lung metastases (A,B).

$R A S$ wt status. Then the patient started a first-line treatment with FOLFIRI (Irinotecan $180 \mathrm{mg} / \mathrm{mq}$ over $90 \mathrm{~min}$ day 1 , calciolevofolinate $200 \mathrm{mg} / \mathrm{mq}$ over $2 \mathrm{~h}$ day $1-2,5$ fluorouracil $400 \mathrm{mg} / \mathrm{mq} \mathrm{3}-4^{\prime}$ day 1,5 fluorouracil $2,400 \mathrm{mg} / \mathrm{mq} 48 \mathrm{~h}$ day 1 every 2 weeks) plus cetuximab ( $400 \mathrm{mg} / \mathrm{mq}$ over $2 \mathrm{~h}$ the first time and then $250 \mathrm{mg} / \mathrm{mq}$ over $1 \mathrm{~h}$ ) for eight cycles with evidence of SD at CT scan and a slight increase of CEA that was $15.6 \mu \mathrm{g} / \mathrm{L}$. Maintenance treatment with weekly cetuximab was then carried out but interrupted after only 2 months when a CT scan showed a progression disease on the lungs where the bigger lesion step from $12 \times 10$ to $15 \times 14 \mathrm{~mm}$ on the inferior left lobe and CEA became $48.5 \mu \mathrm{g} / \mathrm{L}$. Concomitantly we performed a liquid biopsy analysis and $R A S$ and $B R A F$ status were analyzed using a Real-Time PCR method (Easy-KRAS status, EasyNRAS status, Easy-BRAF status, Diatech Pharmacogenetics). From this analysis, a KRAS G13D mutation was identified. In view of this result, KRAS status was determined in the primary tissue of 2015 using the Real-Time PCR assay, revealing a borderline KRAS G13D. These results highlight that the KRAS G13D was already present sub-clonally at the baseline, not identified using the standard mutation analysis methodology, and was responsible for the inefficacy of the anti-EGFR agent. After these evaluations, the patient received a second-line treatment with FOLFOX4 with an SD and improvement of CEA that went down to $10.8 \mu \mathrm{g} / \mathrm{L}$ after five cycles. After ten cycles of treatment an abdominal and chest CT scan showed a dimensional increase of most of the known lung lesions. The patient received Regorafenib (160 mg/die q 21) as third-line treatment for 9 months with stable disease as a better response but a progressive increase of CEA from $35 \mu \mathrm{g} / \mathrm{L}$ to $269 \mu \mathrm{g} / \mathrm{L}$. At progression, Regorafenib was stopped and the patient started Trifluridine/Tipiracil $(700 \mathrm{mg} / \mathrm{mq} \mathrm{q}$ 28) for three cycles, with the radiological progression of disease and CEA increasing to $413 \mu \mathrm{g} / \mathrm{L}$. The patient subsequently received metronomic capecitabine plus hyperthermia with further progression and was referred to palliative care. The patient died about 5 years after the first diagnosis of tumor.

\section{Case C}

In early 2018, a 55 to 60 -years-old patient with persistent constipation and bleeding underwent colonoscopy, with a diagnosis of distal rectal adenocarcinoma with a single synchronous hepatic metastasis (about $25 \mathrm{~mm}$ ) on IV-VIII segment (Figure 3A). CEA was $2.6 \mu \mathrm{g} / \mathrm{L}$ at diagnosis. A pelvic NMR described a rectal cancer extended from $2 \mathrm{~cm}$ above the anal opening to $10 \mathrm{~cm}$, which was defined as cT3N1M1 (Figure 3B). Molecular characterization performed by MassARRAY Sequenom (Myriapod Cancer status, Diatech Pharmacogenetics) of the primary tissue revealed a RAS and BRAF wt status. The patient received a first-line treatment with FOLFOX6 (oxaliplatin $85 \mathrm{mg} / \mathrm{mq}$ over $2 \mathrm{~h}$ day 1, calciolefolinate $200 \mathrm{mg} / \mathrm{mq}$ over $2 \mathrm{~h}$ day 1,5 fluorouracil over $3^{\prime}-4^{\prime}$ day 1,5 fluorouracil over $2400 \mathrm{mg} /$ mq over $48 \mathrm{~h})$ plus panitumumab $(6 \mathrm{mg} / \mathrm{kg})$ every 2 weeks for five cycles and then capecitabine ( $825 \mathrm{mg} / \mathrm{mq}$ BID daily for 6 weeks) with concomitant radiotherapy. At the end of chemoradiotherapy, an abdominal and chest CT scan showed a progression of the liver disease on the IV-VIII segment from $24 \times 20 \mathrm{~mm}$ to $42 \times 36 \mathrm{~mm}$ (Figure 3C) while the pelvic NMR described a regression of the primary tumor (Figure 3D). The pathology report from an anterior resection of the rectum described a complete pathologic regression (pTONOR0) of the primary tumor. It was not possible to perform the liver resection at the same time because of the appearance of a mixed acidosis that would have greatly prolonged the intervention. Therefore, we decided to restart treatment with FOLFOX6 and panitumumab. After 4 cycles, there was an increase of the liver disease up to $55 \times 45 \mathrm{~mm}$ on IV-VIII segment, and the pathologic report from left hepatectomy reported intestinal adenocarcinoma with abundant necrosis. Molecular characterization of the metastasis performed by Next Generation Sequencing (NGS) (Oncomine Focus Assay, Thermofisher Scientific, Monza, Italy) revealed NRAS Q61K and APC c.4199C > A mutations. Liquid biopsy performed at the same time by Real-Time PCR (Easy-NRAS status, Diatech Pharmacogenetics) revealed the presence of NRAS Q61K mutation. These results revealed that in the metastatic lesion, a NRAS N61Q clone was selected and was responsible for the hepatic progression, and liquid biopsy was able to identify such mutation. The repetition of molecular analysis on DNA of the primary tissue, using a Real-Time PCR-based method, revealed a borderline presence of the NRAS Q61Q mutation. This result reinforced the hypothesis that the mutation was present sub-clonally in the primary tissue, but the 

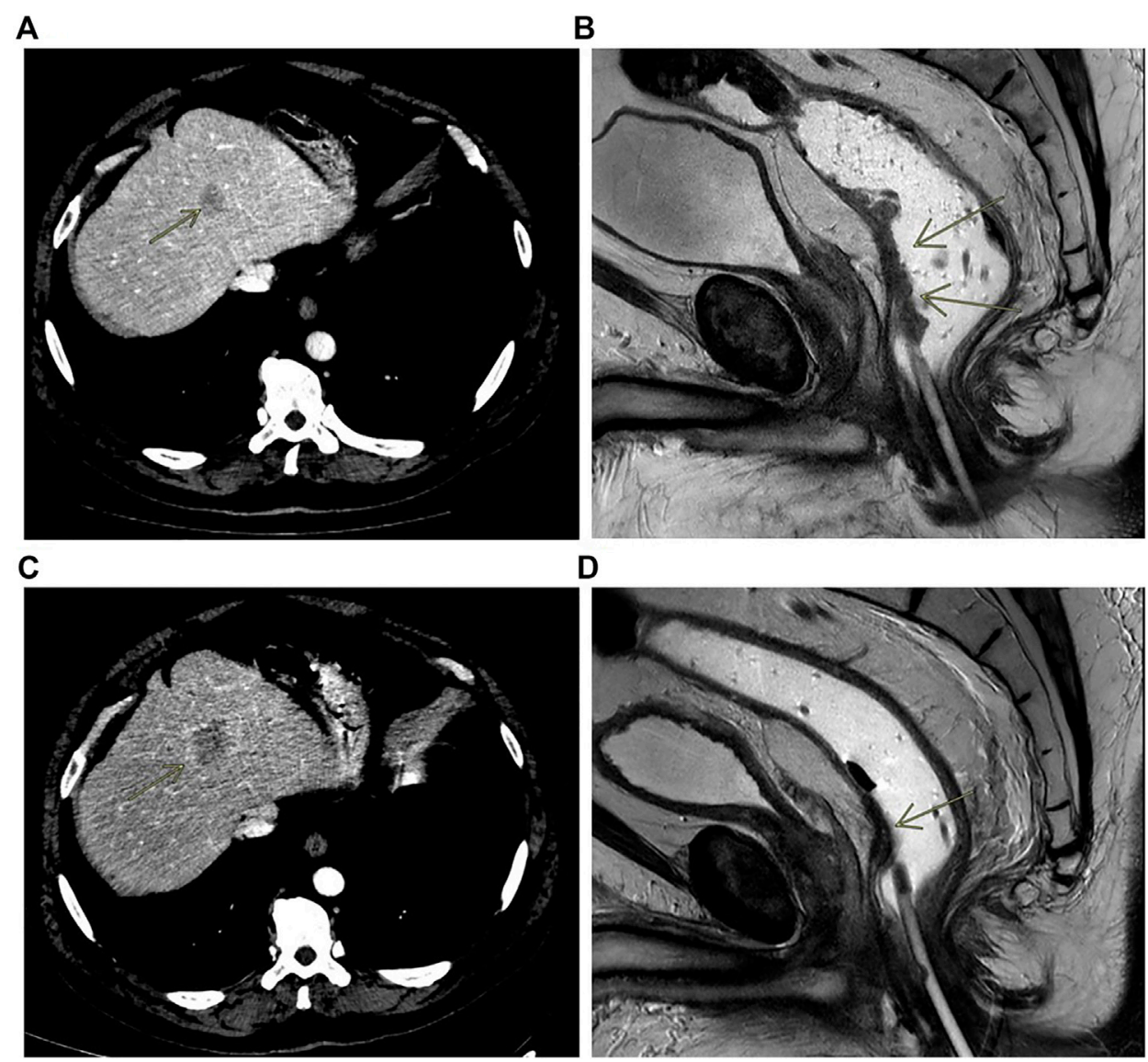

FIGURE 3 | Contrast enhanced CT scan showing liver metastasis at baseline (A) and at progression (C); MRI rectal cancer at baseline (B) and after treatment (D).

low percentage of mutated cells did not obstacle the responsiveness to the anti-EGFR treatment. Conversely, the mutation was selected in the metastatic lesion, rendering it not sensitive to treatment. Liquid biopsy was able to identify the mutation.

An FDG-PET scan performed after liver surgery revealed an early liver and lung progression of disease, so a second-line treatment was started with FOLFIRI (Irinotecan $180 \mathrm{mg} / \mathrm{mq}$ over $90 \mathrm{~min}$ day 1 , calciolevofolinate $200 \mathrm{mg} / \mathrm{mq}$ over $2 \mathrm{~h}$ day $1-2,5$ fluorouracil $400 \mathrm{mg} / \mathrm{mq} 3^{\prime}-4^{\prime}$ day 1,5 fluorouracil $2400 \mathrm{mg} / \mathrm{mq} 48 \mathrm{~h}$ day 1 every 2 weeks) plus bevacizumab ( $5 \mathrm{mg} / \mathrm{kg}$ over $90^{\prime}$ the first time, then $5 \mathrm{mg} / \mathrm{kg}$ over $1 \mathrm{~h} \mathrm{next)}$ every 2 weeks After six cycles of treatment, CEA became positive until $7.2 \mu \mathrm{g} / \mathrm{L}$ and an abdominal and chest CT scan confirmed a progression of disease with multiple lung and liver metastases. Then the patient was treated with 4 cycles of Trifluridina/ Tipiracil (700 mg/mq q 28) but there was again a progression of disease. The patient unfortunately died 2 months after.

\section{Case D}

At the end of 2018, a 45- to 50-year-old patient underwent colonoscopy for abdominal pain with a diagnosis of adenocarcinoma on the right colon. The basal abdominal and chest CT scan described a liver metastasis on V, VI segment about $30 \mathrm{~mm}$ and a doubt lesion about $6 \mathrm{~mm}$ on the superior left lobe of the lung. At diagnosis CEA was $1.6 \mu \mathrm{g} / \mathrm{L}(<5 \mu \mathrm{g} / \mathrm{L})$ and $\mathrm{Ca} 19-914.5 \mathrm{KU} /$ $\mathrm{L}(<37 \mathrm{KU} / \mathrm{L})$. The patient underwent right hemicolectomy and liver resection with a diagnosis of poorly differentiated carcinoma G3 of the colon, with focal aspects of squamous differentiation, and the presence of carcinomatous lymphangitis and hepatic metastasis from poorly differentiated carcinoma. Tissue molecular characterization was performed on specimens of the primary lesion in another center and revealed a RAS and BRAF wt status. Liquid biopsy analysis performed by a Real-Time PCR method (Easy-KRAS status, EasyNRAS status, and Easy-BRAF status, Diatech Pharmacogenetics) confirmed a RAS and BRAF wt status.

In early 2019, the patient received first-line chemotherapy with FOLFOX6 (oxaliplatin $85 \mathrm{mg} / \mathrm{mq}$ over $2 \mathrm{~h}$ day 1 , calciolefolinate $200 \mathrm{mg} / \mathrm{mq}$ over $2 \mathrm{~h}$ day 1,5 fluorouracil over $3^{\prime}-4^{\prime}$ day 1,5 fluorouracil over $2400 \mathrm{mg} / \mathrm{mq}$ over $48 \mathrm{~h}$ ) plus panitumumab $(6 \mathrm{mg} / \mathrm{kg})$ every 2 weeks for five cycles, and the CT scan confirmed a lung metastatic lesion increased to $14 \times 13 \mathrm{~mm}$. An FDG-PET scan confirmed the single lesion. Then the patient was operated on in the superior left lobe of the lung, and the disease was about $15 \mathrm{~mm}$. The diagnosis was poorly differentiated adenocarcinoma, with large necrotic areas compatible with metastases from carcinoma of the large intestine. Upon immunohistochemical investigation, neoplastic cells showed minimal expression of CDX2 and were negative for TTF1, 


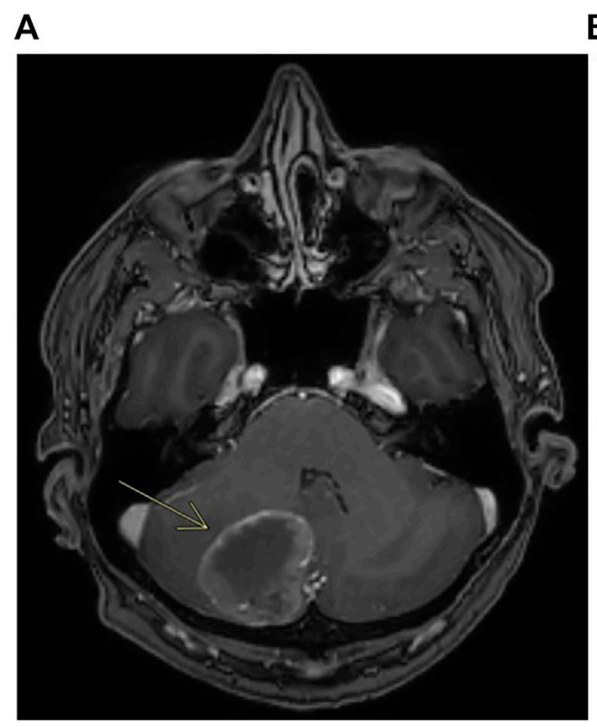

B

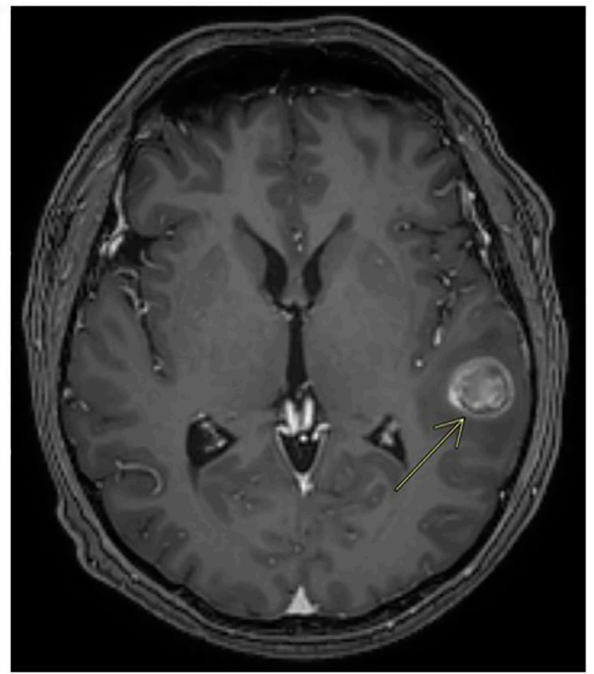

FIGURE 4 | Contrast enhanced MRI showing metastases of the cerebellum (A) and metastases of the left temporal lobe (B).

neuroendocrine markers, and cytokeratins 7 and 20. The growth fraction was $70 \%$. Molecular analysis on this lesion, made using an NGS Focus Oncomine assay (Thermofisher) revealed the presence of PIK3CA c.3073A > G and MAP2K1 c.607G > A mutations. Liquid biopsy performed at the same time using the Oncomine Colon cfDNA Assay (Thermofisher Scientific) revealed the same MAP2K1 mutations, together with an APC mutation (c.4463T > $\mathrm{G})$ that was not covered by the Focus Oncomine assay performed on tissue. It was not possible to verify the presence of the PIK3CA mutation as that specific mutation was not analyzed in the Oncomine Colon cfDNA Assay. After surgery, post-operative chemotherapy with FOLFOX6 was administered. After 4 cycles of treatment an abdominal and chest CT scan did not describe metastatic disease, whereas a brain CT scan performed for referred headache, photophobia and dizziness unfortunately showed a cerebellar lesion about $35 \mathrm{~mm}$ on the right (Figure 4A) and another lesion about $20 \times 20 \mathrm{~mm}$ on temporal left site (Figure $4 \mathrm{~B}$ ).

The patient received craniotomy with cerebellar right resection but the second lesion was not removed. The report was metastatic localization of poorly differentiated adenocarcinoma with large necrotic areas, compatible with known colic primitiveness. Upon immunohistochemical investigation, the cells were only focally positive for CDX2 and negative for CK20 and CK7, TTF1, and Chromogranin. The cell proliferation index, assessed by immunohistochemistry using Ki67/Mib1, was approximately $70 \%$. Molecular analysis of this lesion, made by NGS Oncomine Focus assay, revealed the same spectrum of mutation found in the lung lesion (PIK3CA c.3073A > G, MAP2K1 c.607G > A). At the end of 2019, mediastinal lymph nodes and liver metastases appeared on FDG-PET scan, in the same period the patient received brain tomotherapy (2,500 cGy 5 Fr). In early 2020, a second-line treatment with FOLFIRI (Irinotecan $180 \mathrm{mg} / \mathrm{mq}$ over $90 \mathrm{~min}$ day 1, calciolevofolinate $200 \mathrm{mg} / \mathrm{mq}$ over $2 \mathrm{~h}$ day $1-2,5$ fluorouracil $400 \mathrm{mg} / \mathrm{mq} 3^{\prime}-4^{\prime}$ day 1,5 fluorouracil $2400 \mathrm{mg} / \mathrm{mq} 48 \mathrm{~h}$ day 1 every 2 weeks) was administered for only two cycles but it was stopped because of grade 2 diarrhea and decline of performance status with clinical progression of disease.

By this case report, it has been highlighted that, in this case, liquid biopsy was able to identify the same spectrum of mutation found in tissue, permitting to evidence the presence of mutations carried by the metastatic lesions.

\section{DISCUSSION}

The four patients' histories we reported highlighted the clinical potential utility of liquid biopsy analysis in the management of a patient with advanced CRC. In Case A, we reported a patient with a very aggressive left-sided colorectal tumor that was characterized as $R A S$ and BRAF wt from the tumor molecular analysis. Liquid biopsy analysis performed a few months later revealed the presence of a $B R A F$ V600E mutation in accordance with the highly aggressive tumor behavior (Sanz-Garcia et al., 2017). Considering that the repetition of the analysis on the primary tissue using a more sensitive methodology was not able to reveal the mutation, probably that mutation was present in a heterogeneous manner, unable to be evidenced by a tissue analysis performed on a specific tumor site. Instead, liquid biopsy was able to evidence the mutation, bypassing the problem of heterogeneity. In this case, the knowledge from the beginning of the BRAF V600E mutation would have permitted a different treatment, like FOLFOXIRI and bevacizumab, and in second-line, Binimetinib, Encorafeninb and Cetuximab.

A similar advantage of liquid biopsy was seen for Cases B and C, where the RAS mutation was evidenced by liquid biopsy and not by tumor tissue analysis with standard methodology. In both cases, the repetition of the analysis on tumor tissue with a more sensitive methodology revealed a borderline presence of the mutation. 
These findings again highlighted the heterogeneous presence of the mutation from the beginning, evidenced by liquid biopsy but not by tumor tissue determination. The KRAS G13D mutation in Case $\mathrm{B}$ was the cause of the very low responsiveness to anti-EGFR therapy, and knowledge of the mutation status from the beginning would have indicated an alternative treatment for the patient. Similarly, the NRAS N61Q mutation in Case $C$ was evident in the metastatic lesion at relapse and in the liquid biopsy, but was present at borderline and evidenced only using very sensitive methodologies in the primary tumor tissue. In addition, in this case, the knowledge of the mutation would have enabled treating the patient with an alternative treatment strategy. Probably we would have used anti-VEGF in association to chemotherapy upfront and over progression.

The last case we reported (Case D) highlighted that gene alterations other than RAS and BRAF could be responsible for the unresponsiveness to anti-EGFR agents. In fact, $P I K 3 C A$ and $M A P 2 K 1$ mutations were seen in the metastatic lesions and liquid biopsy. Although it was not possible to verify the presence of such mutations from the beginning, as a targeted sequencing was performed instead of an NGS approach, we observed that the mutation was determinable in liquid biopsy.

Different methodologies are used for RAS and BRAF determinations, having different sensitivity of detection ranging from 0.1 to 5\% (Tan and Du, 2012; Taniguchi et al., 2015). Many molecular diagnostic laboratories do not routinely use ultrasensitive methodologies for tumor tissue molecular characterization, and some under-represented mutations could be missed. This is true, in particular, if the mutation is heterogeneously present within the tumor (Gargalionis and Papavassiliou, 2017; Khan et al., 2018; Molinari et al., 2018). Liquid biopsy analysis could represent an optimal option, representing the overall tumor spectrum mutation.

Another important advantage of liquid biopsy is the possibility to monitor and identify mutations that will arise from the primary tumor to the metastatic lesion (Siravegna et al., 2015). Several reports have demonstrated the possibility to monitor, through liquid biopsy, the arising of resistance mutations during treatment with anti-EGFR therapies (Gargalionis and Papavassiliou, 2017; Khan et al., 2018; Chen et al., 2019; Kastrisiou et al., 2019).

We have demonstrated that NGS analysis on liquid biopsies could be feasible and could permit us to analyze concomitantly different gene mutations, permitting us to evidence new alterations arising during treatment that could potentially be the target of subsequent targeted treatments. A recent study showed that liquid biopsy analysis allowed an increase in therapeutic options in one third of analyzed patients (Pereira et al., 2020). Moreover, clinical trials have been performed in which liquid biopsy was used to drive clinical decisions. In a phase II trial designed by Cremolini et al. it was demonstrated that a challenging strategy with cetuximab and irinotecan may be active in patients with RAS and BRAF wt mCRC with acquired resistance to first-line irinotecan- and cetuximab-based therapy. The evaluation of RAS mutational status on ctDNA might be helpful in selecting candidate patients.

The increasing consolidation of the use of NGS methodologies in liquid biopsy has opened the possibility to monitor multiple gene alterations, with potentialities in monitoring response to treatment and in identifying emerging resistance mutations (Kastrisiou et al., 2019).
Despite the potential of NGS on liquid biopsy in mCRC, the specific limitations have to be considered. The principal limitation is sensitivity. The issue of sensitivity is highly relevant in the context of liquid biopsies, where mutant ctDNA is like a "needle in a haystack" of wt DNA fragments. Some other high sensitive methodologies have been proposed for evaluation of mutations in liquid biopsies, such as digital droplet PCR (Ono et al., 2017; Decraene et al., 2018) also applied to extracellular vesicles (Notarangelo et al., 2019). However, the increasing knowledge of tumor mutations and the increase of targeted therapeutic drugs available render NGS analysis more suitable for a wide molecular characterization. In the future, the development of NGS platforms with higher sensitivity will further upgrade the study of tumor heterogeneity in the blood. The use of targeted NGS to monitor these mutations in real-time may set the foundation for a new approach in the management of cancer.

\section{CONCLUSION}

By these four clinical case reports we highlighted that liquid biopsy could have a role in the management of mCRC patients, giving complementary information that could be very important for the treatment decision making. Tumor heterogeneity and its dynamic evolution over time are both aspects that could affect the reliability of tumor tissue analysis. Although no indications are present for the use of liquid biopsy in the clinical management of mCRC patients, clinicians could consider it as an option in specific circumstances. The test is rapid and with a relatively low cost, both aspects that facilitate its use in the clinical practice. There is currently no indication to administer targeted drugs (outside of clinical trials) based on mutations identified by liquid biopsy, but it is desirable that this can happen in the near future to expand the therapeutic opportunities of our patients.

\section{DATA AVAILABILITY STATEMENT}

The datasets presented in this article are not readily available due to ethical and privacy restrictions. Requests to access the datasets should be directed to the corresponding author.

\section{ETHICS STATEMENT}

Ethical approval was obtained from the Institutional Research Ethics Committee (C.E.ROM IRSTB038). The patients/participants provided their written informed consent to participate in this study.

\section{AUTHOR CONTRIBUTIONS}

PU, AP, GM, and MM made substantial contributions to the conception, design, and interpretation of the data. GM, EC, CM, MC, LP, FF, GF, and GB performed the experiments and carried out the formal analysis of the data. PU, AP, GM, and MM wrote the initial draft of the manuscript, and reviewed and edited it. All authors read and approved the manuscript. 


\section{REFERENCES}

Chen, S. H., Tsai, H. L., Jiang, J. K., Sung, Y. C., Huang, C. W., Yeh, Y. M., et al. (2019). Emergence of RAS Mutations in Patients with Metastatic Colorectal Cancer Receiving Cetuximab-Based Treatment: a Study Protocol. BMC Cancer 19, 640. doi:10.1186/s12885-019-5826-7

Decraene, C., Silveira, A. B., Bidard, F. C., Vallée, A., Michel, M., Melaabi, S., et al. (2018). Multiple Hotspot Mutations Scanning by Single Droplet Digital PCR. Clin. Chem. 64, 317-328. doi:10.1373/clinchem.2017.272518

Di Fiore, F., Blanchard, F., Charbonnier, F., Le Pessot, F., Lamy, A., Galais, M. P., et al. (2007). Clinical Relevance of KRAS Mutation Detection in Metastatic Colorectal Cancer Treated by Cetuximab Plus Chemotherapy. Br. J. Cancer 96, 1166-1169. doi:10.1038/sj.bjc.6603685

Diehl, F., Schmidt, K., Choti, M. A., Romans, K., Goodman, S., Li, M., et al. (2008). Circulating Mutant DNA to Assess Tumor Dynamics. Nat. Med. 14, 985-990. doi:10.1038/nm.1789

Furuki, H., Yamada, T., Takahashi, G., Iwai, T., Koizumi, M., Shinji, S., et al. (2018). Evaluation of Liquid Biopsies for Detection of Emerging Mutated Genes in Metastatic Colorectal Cancer. Eur. J. Surg. Oncol. 44, 975-982. doi:10.1016/j.ejso.2018.01.224

Gargalionis, A. N., and Papavassiliou, A. G. (2017). Liquid Biopsies in Colorectal Cancer: Monitoring Genetic Heterogeneity. Trends Cancer 3, 166-168. doi:10.1016/j.trecan.2017.01.003

Karapetis, C. S., Khambata-Ford, S., Jonker, D. J., O'Callaghan, C. J., Tu, D., Tebbutt, N. C., et al. (2008). K-ras Mutations and Benefit from Cetuximab in Advanced Colorectal Cancer. N. Engl. J. Med. 359, 1757-1765. doi:10.1056/NEJMoa0804385

Kastrisiou, M., Zarkavelis, G., Pentheroudakis, G., and Magklara, A. (2019). Clinical Application of Next-Generation Sequencing as A Liquid Biopsy Technique in Advanced Colorectal Cancer: A Trick or A Treat? Cancers (Basel) 11, 1573. doi:10.3390/cancers11101573

Khan, K. H., Cunningham, D., Werner, B., Vlachogiannis, G., Spiteri, I., Heide, T., et al. (2018). Longitudinal Liquid Biopsy and Mathematical Modeling of Clonal Evolution Forecast Time to Treatment Failure in the PROSPECT-C Phase II Colorectal Cancer Clinical Trial. Cancer Discov. 8, 1270-1285. doi:10.1158/2159-8290.CD-17-0891

Knijn, N., Mekenkamp, L. J., Klomp, M., Vink-Börger, M. E., Tol, J., Teerenstra, S., et al. (2011). KRAS Mutation Analysis: a Comparison between Primary Tumours and Matched Liver Metastases in 305 Colorectal Cancer Patients. Br. J. Cancer 104, 1020-1026. doi:10.1038/bjc.2011.26

Misale, S., Yaeger, R., Hobor, S., Scala, E., Janakiraman, M., Liska, D., et al. (2012). Emergence of KRAS Mutations and Acquired Resistance to Anti-EGFR Therapy in Colorectal Cancer. Nature 486, 532-536. doi:10.1038/nature11156

Molinari, C., Marisi, G., Passardi, A., Matteucci, L., De Maio, G., and Ulivi, P. (2018). Heterogeneity in Colorectal Cancer: A Challenge for Personalized Medicine? Int. J. Mol. Sci. 19, 3733. doi:10.3390/ijms19123733

Murtaza, M., Dawson, S. J., Tsui, D. W., Gale, D., Forshew, T., Piskorz, A. M., et al. (2013). Non-invasive Analysis of Acquired Resistance to Cancer Therapy by Sequencing of Plasma DNA. Nature 497, 108-112. doi:10.1038/nature12065

Notarangelo, M., Zucal, C., Modelska, A., Pesce, I., Scarduelli, G., Potrich, C., et al. (2019). Ultrasensitive Detection of Cancer Biomarkers by Nickel-Based
Isolation of Polydisperse Extracellular Vesicles from Blood. EBioMedicine 43, 114-126. doi:10.1016/j.ebiom.2019.04.039

Ono, Y., Sugitani, A., Karasaki, H., Ogata, M., Nozaki, R., Sasajima, J., et al. (2017). An Improved Digital Polymerase Chain Reaction Protocol to Capture LowCopy KRAS Mutations in Plasma Cell-free DNA by Resolving 'subsampling' Issues. Mol. Oncol. 11, 1448-1458. doi:10.1002/1878-0261.12110

Pereira, J., Alves, F., Ferreira, F., Vasconcelos de Matos, L., Massena, A., and Martins, A. (2020). Liquid Biopsies in Progressing Metastatic Colorectal Cancer- Application and Their Therapeutic Implications According to the RAS Status. Cureus. 12, e7035. doi:10.7759/cureus.7035

Sanz-Garcia, E., Argiles, G., Elez, E., and Tabernero, J. (2017). BRAF Mutant Colorectal Cancer: Prognosis, Treatment, and New Perspectives. Ann. Oncol. 28, 2648-2657. doi:10.1093/annonc/mdx401

Siravegna, G., Mussolin, B., Buscarino, M., Corti, G., Cassingena, A., Crisafulli, G., et al. (2015). Clonal Evolution and Resistance to EGFR Blockade in the Blood of Colorectal Cancer Patients. Nat. Med. 21, 795-801. doi:10.1038/nm.3870

Takeda, K., Yamada, T., Takahashi, G., Iwai, T., Ueda, K., Kuriyama, S., et al. (2019). Analysis of Colorectal Cancer-Related Mutations by Liquid Biopsy: Utility of Circulating Cell-free DNA and Circulating Tumor Cells. Cancer Sci. 110, 3497-3509. doi:10.1111/cas.14186

Tan, C., and Du, X. (2012). KRAS Mutation Testing in Metastatic Colorectal Cancer. World J. Gastroenterol. 18, 5171-5180. doi:10.3748/wjg.v18.i37.5171

Taniguchi, H., Yamazaki, K., Yoshino, T., Muro, K., Yatabe, Y., Watanabe, T., et al. (2015). Japanese Society of Medical Oncology Clinical Guidelines: RAS (KRAS/ NRAS) Mutation Testing in Colorectal Cancer Patients. Cancer Sci. 106, 324-327. doi:10.1111/cas.12595

Yamada, T., Iwai, T., Takahashi, G., Kan, H., Koizumi, M., Matsuda, A., et al. (2016). Utility of KRAS Mutation Detection Using Circulating Cell-free DNA from Patients with Colorectal Cancer. Cancer Sci. 107, 936-943. doi:10.1111/ cas. 12959

Conflict of Interest: The authors declare that the research was conducted in the absence of any commercial or financial relationships that could be construed as a potential conflict of interest.

Publisher's Note: All claims expressed in this article are solely those of the authors and do not necessarily represent those of their affiliated organizations, or those of the publisher, the editors and the reviewers. Any product that may be evaluated in this article, or claim that may be made by its manufacturer, is not guaranteed or endorsed by the publisher.

Copyright (c) 2021 Ulivi, Passardi, Marisi, Chiadini, Molinari, Canale, Pasini, Ferroni, Frassineti, Bartolini and Monti. This is an open-access article distributed under the terms of the Creative Commons Attribution License (CC $B Y)$. The use, distribution or reproduction in other forums is permitted, provided the original author(s) and the copyright owner(s) are credited and that the original publication in this journal is cited, in accordance with accepted academic practice. No use, distribution or reproduction is permitted which does not comply with these terms. 